\title{
Etnobotánica y derechos de la naturaleza en el aja shuar: caso de estudio parroquia Nankais, cantón Nangaritza, provincia Zamora Chinchipe, Ecuador
}

\author{
Ethnobotany and rights of nature in the aja shuar: \\ case study Nankais parish, Nangaritza canton, Zamora \\ Chinchipe province, Ecuador
}

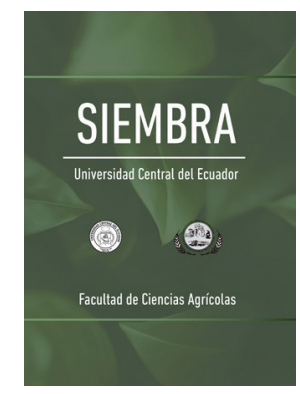

Hector Rolando Zhiñin Quezada ${ }^{1}$, Bladimir Valentin Poma Mendoza ${ }^{2}$, Leonardo Paúl González Nivelo ${ }^{3}$, Gonzalo Bladimir Quito Ulloa ${ }^{4}$

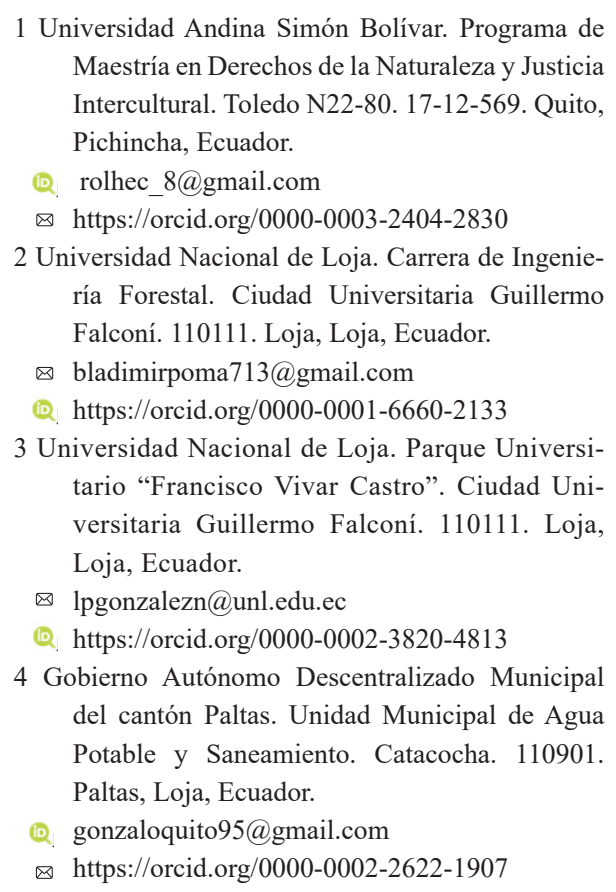

1 Universidad Andina Simón Bolívar. Programa de Maestría en Derechos de la Naturaleza y Justicia Intercultural. Toledo N22-80. 17-12-569. Quito, Pichincha, Ecuador.

(D) rolhec_8@gmail.com

$凶$ https://orcid.org/0000-0003-2404-2830

2 Universidad Nacional de Loja. Carrera de Ingeniería Forestal. Ciudad Universitaria Guillermo Falconí. 110111. Loja, Loja, Ecuador.

凶bladimirpoma713@gmail.com

(D. https://orcid.org/0000-0001-6660-2133

3 Universidad Nacional de Loja. Parque Universitario "Francisco Vivar Castro". Ciudad Universitaria Guillermo Falconí. 110111. Loja, Loja, Ecuador.

凶1pgonzalezn@unl.edu.ec

(C) https://orcid.org/0000-0002-3820-4813

4 Gobierno Autónomo Descentralizado Municipal del cantón Paltas. Unidad Municipal de Agua Potable y Saneamiento. Catacocha. 110901. Paltas, Loja, Ecuador.

(D) gonzaloquito95@gmail.com

$凶$ https://orcid.org/0000-0002-2622-1907

*Autor de correspondencia: rolhec.8@gmail.com

\section{SIEMBRA}

https://revistadigital.uce.edu.ec/index.php/SIEMBRA ISSN-e: $2477-8850$

ISSN: $1390-8928$

Periodicidad: semestral

vol. 8, núm. 2, 2021

siembra.fag@uce.edu.ec

DOI: https://doi.org/10.29166/siembra.v8i2.3036

Esta obra está bajo una licencia internacional Creative Commons Atribución-NoComercial

\section{Resumen}

La Constitución de 2008 del Ecuador reconoce a la naturaleza como titular de derechos; en este contexto, las comunidades que aún conservan sus saberes ancestrales y ecosistemas están experimentando procesos de aculturación, con escasa documentación etnobotánica, lo que implica una pérdida progresiva de la biodiversidad y calidad de vida. Con estos antecedentes el presente estudio pretende realizar un análisis sobre los derechos de la naturaleza y la etnobotánica de siete ajas shuar en la parroquia Nankais, región sur de Ecuador. Con este fin se realizaron talleres participativos y entrevistas con las informantes, para documentar la etnobotánica y manejos realizados en el aja; así también, se realizó el inventario de las ajas (20 ha) para identificar in situ las especies utilizadas por la comunidad. Adicionalmente, se efectuó un análisis basado en el ordenamiento jurídico con énfasis en el derecho de las comunidades de conservar y promover sus prácticas de manejo de la biodiversidad y su entorno natural. Se reportaron 67 especies, 57 géneros y 33 familias botánicas. Las familias más representativas fueron Arecaceae y Solanaceae; se estableció que 20 especies tienen usos medicinales y 18 especies se emplean en la alimentación. Se resalta que la comunidad se beneficia de manera armónica del aprovechamiento de productos forestales no madereros -PFNM(sumak kawsay) y aplica técnicas de manejo basadas en conocimientos ecológicos tradicionales compatibles con el derecho primordial de la naturaleza a su existencia, el mantenimiento y regeneración de sus ciclos vitales, estructura, funciones y procesos evolutivos consagrados en el ordenamiento jurídico ecuatoriano e internacional.

Palabras clave: aculturación, diversidad biológica, etnobotánica, conocimientos tradicionales.

\section{Abstract}

The 2008 Constitution of Ecuador recognizes nature as a holder of rights, in this context the communities that still preserve their 
ancestral knowledge and ecosystems are experiencing acculturation processes, with little ethnobotanical documentation, which implies a progressive loss of biodiversity and quality of life. With this background, the present study aims to conduct an analysis between the rights of nature and the ethnobotany of seven Shuar Ajas in the Nankais Parish, southern region of Ecuador. Thus, participatory workshops and interviews with the informants were conducted to document the ethnobotany and management carried out in the aja, as well as an inventory of the Ajas (20 ha) to identify in situ the species used by the community. In addition, an analysis was carried out based on the legal system with emphasis on the right of the communities to conserve and promote their biodiversity management practices and their natural environment. A total of 67 species, 57 genera and 33 botanical families were reported. The most representative families were Arecaceae and Solanaceae. It was recorded that 20 species have medicinal uses and 18 species are for food use. It should be noted that the community benefits harmoniously from the use of NTFPs (Sumak Kawsay) and applies management techniques based on traditional ecological knowledge compatible with the primordial right of nature to its existence, maintenance and regeneration of its life cycles, structure, functions and evolutionary processes enshrined in the Ecuadorian and international legal system.

Keywords: acculturation, biological diversity, ethnobotany, traditional knowledge.

\section{Introducción}

El marco constitucional ecuatoriano reconoce a la naturaleza como sujeto de derechos y garantiza a las comunas, comunidades, pueblos y nacionalidades indígenas varios derechos colectivos, entre ellos el de conservar y promover sus prácticas de manejo de la biodiversidad y de su entorno natural en el cual se desarrollan (Constitución del Ecuador, 2008). En este ámbito encontramos a las aja shuar, las cuales son definidas por las mujeres shuar como "Un ser, una mujer donde tú tienes todo, traes a los niños y tú mismo te diviertes. Mi aja es mi mejor tesoro, si no tengo aja, soy doña nada. La aja es una fuente de mercado" (Reátegui Encarnación, 2011, p. 30).

En este contexto, la región amazónica de Ecuador se caracteriza por poseer gran biodiversidad en sus cuatro componentes: especies, genes, ecosistemas y los saberes ancestrales, por parte de las diferentes comunidades nativas que se desenvuelven en áreas boscosas nativas (Instituto Nacional de Investigaciones Agropecuarias [INIAP], 2008), este grupo importante realiza prácticas silviculturales sostenibles desde tiempos inmemorables (Grijalva et al., 2013), ya que tienen como prioridad el uso alternativo del bosque en productos alimenticios para su subsistencia, lo cual implica un importante conocimiento del bosque, sus recursos y usos (Santín Luna, 2004).

Específicamente, en la llamada alta Amazonía o selva alta ecuatoriana, en las actuales provincias de Zamora Chinchipe y Morona Santiago al sur de la provincia de Pastaza (Aij', 1984), se encuentra la nacionalidad shuar que, etimológicamente, significa "gente" o "persona", son la etnia más importante del país; con lenguaje, costumbres y formas de vida propias, es decir, una cultura única. Son el pueblo amazónico más numeroso (aproximadamente 110.000 individuos). Su idioma oficial es el shuar chicham, y poseen una gran riqueza cultural y religiosa, cuya principal filosofía es el respeto a la naturaleza; no obstante, en los últimos tiempos han perdido su identidad por influencias de otras culturas y por el rechazo social (Balcázar Calle, 2016; Gálvez, 2010).

En la provincia de Zamora Chinchipe, cantón Nangaritza, parroquia rural de Nankais, el 86 \% de su población está representada por la nacionalidad shuar, la misma que maneja zonas en las cuales aún se conservan áreas de bosque casi inalteradas, y ven al bosque y el aja como su forma de vida y tratan de conservarlos; éstos dependen del ambiente natural para vivir y especialmente de las plantas, puesto que les dan diferentes usos: medicinal, comestible, artesanal, alucinógeno, entre otros (Santín Luna, 2004).

Desde la visión occidental, se entiende como aja shuar, una huerta de cultivos, no obstante, para la nacionalidad shuar es el espacio donde se plasma y desarrolla los saberes y prácticas ancestrales; dicho en otras palabras, es donde se fundamenta la cosmovisión shuar (Reátegui Encarnación, 2011). Así, por iniciativa de un grupo de lideresas shuar preocupadas por conservar los saberes y prácticas ancestrales del aja, surgió el presente estudio, el cual constató que el aja alberga una gran diversidad de especies vegetales las cuales se aprovechan en diferentes actividades cotidianas como: musicales (instrumentos), dancísticas (festival de la chonta), gastronómicas (chicha) y espirituales (rituales de natem).

Los productos forestales no madereros (PFNM) han contribuido al bienestar y progreso de la parroquia Nankais (sumak kawsay) (Gobierno Autónomo Descentralizado [GAD] Nankais, 2019), así como a la preser- 
vación del conocimiento profundo sobre la diversidad de usos y beneficios que tiene el bosque como medicina tradicional, alimentación y artesanías; esto ha motivado al shuar a conservar sus ecosistemas con una complejidad alta. Para ello, cada año la familia integra una aja nueva al sistema, con riqueza y diversidad de especies nativas que se aprovechan con frecuencias mínimas, puesto que la fenología de las plantas se presenta en distintas épocas del año, así también las intensidades y volúmenes de cosecha son bajas, ya que la producción es para subsistencia familiar. Cabe destacar las ajas tienen un ciclo de aprovechamiento de dos a tres años y luego pasarían a periodo barbecho (Reátegui Encarnación, 2011).

Finalmente, el enfoque de la etnobotánica bajo la luz de los derechos de la naturaleza, implica el respeto hacia la diversidad, los procesos de interrelación, autopoiesis de los ecosistemas y el conocimiento tradicional de las comunidades, pueden contribuir considerablemente en la planificación del desarrollo integrado y manejo adecuado de los recursos naturales. Con estos antecedentes el presente estudio pretende realizar un análisis de la etnobotánica de siete ajas shuar, bajo la luz de los derechos de la naturaleza, con énfasis en el sumak kawsay (buen vivir) en la parroquia Nankais, Región Sur de Ecuador (RSE).

\section{Materiales y métodos}

\section{1. Área de estudio}

La parroquia rural de Nankais se encuentra ubicada en el sur del Ecuador, en la provincia de Zamora Chinchipe, al noreste del cantón Nangaritza (Figura 1), tiene una superficie de $7.700 \mathrm{~km}^{2}$, su población es de 775 habitantes, siendo el mayor porcentaje de población indígena con un $86 \%$, pertenecientes en su totalidad al grupo étnico shuar, su altitud es de $850 \mathrm{~m}$ s.n.m. con un clima subtropical cálido y una temperatura promedio de $25^{\circ} \mathrm{C}$ (GAD Nankais, 2019).

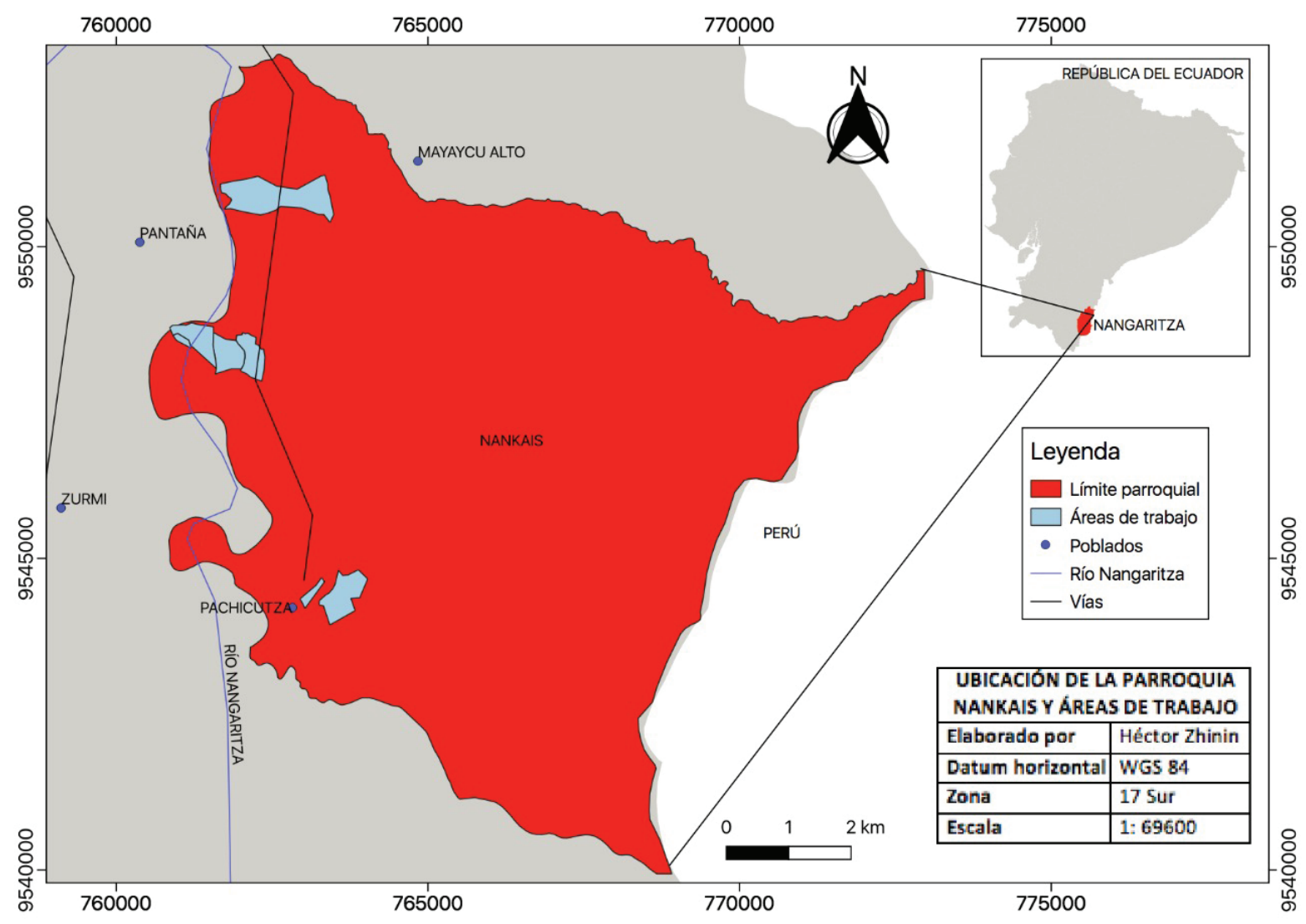

Figura 1. Mapa de ubicación de la parroquia Nankais y áreas de trabajo (Nangaritza-Zamora Chinchipe-RSE). Figure 1. Location map of the Nankais parish and work areas (Nangaritza-Zamora Chinchipe-RSE) 


\subsection{Estudio etnobotánico}

La información etnobotánica se recopiló durante el mes de diciembre de 2020, mediante la aplicación de tres técnicas de investigación social-cualitativas de recopilación:

- Talleres participativos. Se partió por el taller en el cual se recopiló las especies vegetales útiles, elementos botánicos recolectados, así como los nombres comunes y algunas características exomorfológicas.

- Entrevistas semiestructuradas. A través de estas entrevistas se indagó acerca de las aplicaciones de las plantas reportadas.

- Observación. Se aplicó para contrastar la presencia de las especies reportadas por las informantes en los talleres participativos, así como también para documentar las prácticas de manejo y los vínculos culturales y espirituales establecidos, a partir de sus interacciones con los recursos naturales.

\subsection{Caracterización de las informantes}

Se trabajó con siete mujeres de la nacionalidad shuar (informantes), perteneciente a un grupo etario de 30-50 años, lideresas y activistas por la conservación de los saberes ancestrales de los barrios: San Manuel (2), Tsarunts (3) y Pachkius (2) de la parroquia Nankais, cabe destacar que estas mujeres consideran estos espacios como respetables y mantienen una interconexión íntima con los mismos, puesto que la consideran como un todo y su estilo de vida donde se fundamenta la cosmovisión shuar.

Cabe mencionar que estas lideresas se encargan del manejo de sus propias ajas, en consecuencia, han adquirido una infinidad de conocimientos relacionados con las formas de empleo, usos, formas de preparación, aplicaciones de las plantas y los nombres comunes. Así, motivados por voluntades propias, colaboraron con la investigación con la convicción de empoderar a los demás miembros de su comunidad y hacer conocer su lucha y cultura.

\subsection{Diversidad especifica}

Se aplicó la metodología propuesta por Aguirre (2012), en la cual se determina la diversidad específica mediante el número de especies, ya que es la medida más frecuentemente utilizada, debido a que la riqueza de especies refleja distintos aspectos de la biodiversidad y, tanto el concepto de especie como su significado, son ampliamente entendidos, son fácilmente detectables y cuantificables, y se cuenta con un sinnúmero de datos disponibles sobre el número de especies (información secundaria).

Con esta base, para su identificación se realizó trabajo de campo que consistió en recorridos en las ajas con el acompañamiento de las mujeres shuar, para colectar muestras botánicas fértiles de especies identificadas como útiles, de acuerdo con las categorías de uso propuestas por Morales Valverde et al. (2011), las mismas que se modificaron acorde a los resultados obtenidos (Tabla 1).

La determinación taxonómica de las muestras botánicas se realizó en el herbario Reinaldo Espinosa de la Universidad Nacional de Loja, luego se contrastó con bases de datos taxonómicas de especies florísticas probadas y confiables como: Trópicos, Base Nacional de Datos de Biodiversidad del Ecuador (BNDB) y The Plant List (TPL).

Tabla 1. Categorías utilizadas para clasificar los usos en el estudio.

Table 1. Categories used to classify the uses in the study.

Categoría de uso

Definición

\begin{tabular}{ll}
\hline Alimentación humana & $\begin{array}{l}\text { Incluidas bebidas (licores, infusiones, refrescantes, etc.), condimentos, conservantes, aromatizantes, colorantes, arro- } \\
\text { pías (caramelos), masticatorias, frutos secos), oleaginosas (alimentarias), etc. }\end{array}$ \\
\hline $\begin{array}{l}\text { Medicinales, } \\
\text { veterinarias y tóxicas }\end{array}$ & $\begin{array}{l}\text { Se incluyen aquí todas las plantas con cualquier uso y propiedad medicinal o tóxica para el hombre y sus ganados, } \\
\text { utilizadas tanto en medicina tradicional y herboristería como en la industria farmacéutica, así como las empleadas en la } \\
\text { preparación de cualquier tipo de medicamentos (excipientes, tinturas, jarabes, etc.). Se incluyen también las fumatorias } \\
\text { y repelentes de animales (insecticidas, raticidas) }\end{array}$ \\
\hline $\begin{array}{l}\text { Industriales } \\
\text { y artesanales }\end{array}$ & $\begin{array}{l}\text { Incluye todo tipo de usos de esta naturaleza, como por ejemplo: perfumeras, cosméticas, textiles, tintóreas, maderables, } \\
\text { cestería, juguetería, instrumentos musicales, aperos y herramientas, resinosas, leñas y combustibles, carboneo, lejías, etc. }\end{array}$ \\
\hline Folclore & Plantas ceremoniales, ritos religiosos, fiestas, magia, hechicería, etc. \\
\hline
\end{tabular}

Nota: Adaptado de Biodiversidad y etnobotánica en España de Morales Valverde et al. (2011) 


\subsection{Análisis estadístico}

Se analizó descriptivamente el porcentaje de las familias y especies más representativas dentro de cada una de las categorías. Para determinar el valor cultural de una especie se utilizó el Índice de valor de uso (IVUs) (ecuación [1]) aplicado por Cogollo Calderón y García-Cossio (2012), que se establece de acuerdo con el grado de consenso en las respuestas de los informantes, con respecto a la utilidad de una especie. Este índice se estimó utilizando la expresión:

$$
I V U S=\frac{\Sigma U V i s}{N s}
$$

donde:

- $U V i s=$ número de usos mencionados por cada informante (i), para cada especie.

- $N s=$ número de informantes entrevistados.

\subsection{Base normativa}

Se realizó un análisis de las disposiciones constitucionales sobre el reconocimiento de los derechos de la naturaleza (mura = bosque), artículo 74 de la Constitución del Ecuador: "Las personas, comunidades, pueblos y nacionalidades tendrán derecho a beneficiarse del ambiente y de las riquezas naturales que les permitan el buen vivir" (Constitución de la República del Ecuador, 2008), el sumak kawsay (arts. 14 y 15) el cual propone un vínculo de armonía con la naturaleza, considerando que todos pertenecen a un mismo conjunto (Figuera Vargas y Cujilema Quinchuela, 2017).

El Convenio 169 sobre pueblos indígenas y tribales (1989), establece que los pueblos indígenas y tribales deberán tener el derecho a decidir sus propias prioridades en lo que atañe al proceso de desarrollo, creencias, instituciones y bienestar espiritual y a las tierras que ocupan o utilizan de alguna manera (Oficina Internacional del Trabajo [OIT]., 2007). Así también, se incorporaron elementos de los derechos bioculturales de las comunidades conducentes a mantener, proteger y controlar su cultura y sus conocimientos ecológicos tradicionales.

\section{Resultados}

\subsection{Composición y riqueza florística}

Se identificaron 33 familias botánicas, representadas en 57 géneros y 67 especies. La familia más empleada corresponde a Arecaceae con 7 géneros y 7 especies (Bactris gasipaes, Desmoncus sp., Euterpe precatoria, Iriartea deltoidea, Mauritia flexuosa, Oenocarpus bataua, Wettinia maynensis), seguida de Solanaceae con 4 géneros y 7 especies (Tabla 2 y Figura 2).

Tabla 2. Composición florística de los PFNM de origen vegetal en siete ajas shuar de la parroquia Nankais.

Table 2. Floristic composition of NTFPs of plant origin in seven shuar ajas of the Nankais parish.

\begin{tabular}{|c|c|c|c|c|c|}
\hline Categoría & Nombre científico & Nombre común & Familia botánica & IVUs* & $\mathrm{HC}^{* *}$ \\
\hline \multirow{11}{*}{$\begin{array}{l}\text { Alimentación } \\
\text { humana }\end{array}$} & Arachis hypogaea $\mathrm{L}$. & Maní & Fabaceae & 0,29 & Hierba \\
\hline & Bactris gasipaes Kunth & Chonta. uwi & Arecaceae & 0,43 & Palma \\
\hline & Batocarpus orinocensis H. Karst. & Pitiu & Moraceae & 0,14 & Árbol \\
\hline & Colocasia esculenta (L.) Schott & Papa china & Araceae & 0,29 & Hierba \\
\hline & Euterpe precatoria Mart. & Palmito & Arecaceae & 0,43 & Palma \\
\hline & Grias peruviana Miers & Apay & Lecythidaceae & 0,14 & Árbol \\
\hline & Inga edulis Mart. & Guaba bejuco & Fabaceae & 0,14 & Árbol \\
\hline & Inga spectabilis (Vahl) Willd. & Guaba machetona & Fabaceae & 0,14 & Árbol \\
\hline & Ipomoea batatas (L.) Lam. & Camote & Convolvulaceae & 0,14 & Rastrero \\
\hline & Manihot esculenta Crantz & Yuca & Euphorbiaceae & 0,43 & Arbusto \\
\hline & Mauritia flexuosa L.f. & Hacho, morete & Arecaceae & 0,43 & Palma \\
\hline
\end{tabular}




\begin{tabular}{|c|c|c|c|c|c|}
\hline \multirow{14}{*}{$\begin{array}{l}\text { Alimentación } \\
\text { humana }\end{array}$} & Persea americana Mill. & Aguacate & Lauraceae & 0,29 & Árbol \\
\hline & Physalis pubescens $\mathrm{L}$. & Uvilla & Solanaceae & 0,29 & Hierba \\
\hline & Plukenetia volubilis $\mathrm{L}$. & Maní de monte & Euphorbiaceae & 0,86 & Arbusto \\
\hline & Pourouma cecropiifolia Mart. & Uva de monte & Urticaceae & 0,14 & Árbol \\
\hline & Pourouma minor Benoist & Uva de monte & Urticaceae & 0,14 & Árbol \\
\hline & Pouteria caimito (Ruiz y Pav.) Radlk. & Yarazo & Sapotaceae & 0,14 & Árbol \\
\hline & Psidium guajava $\mathrm{L}$. & Guayaba & Myrtaceae & 1 & Árbol \\
\hline & Quararibea cordata (Bonpl.) Vischer & Zapote & Malvaceae & 0,29 & Árbol \\
\hline & Renealmia sp. & Kumpia & Zingiberaceae & 0,14 & Hierba \\
\hline & Smallanthus sp. & Jícama & Asteraceae & 0,14 & Arbusto \\
\hline & Solanum sessiliflorum Dunal & Naranjilla cocona & Solanaceae & 0,14 & Arbusto \\
\hline & Solanum stramoniifolium Jacq. & Yakukuck & Solanaceae & 0,14 & Arbusto \\
\hline & Theobroma cacao L. & Cacao & Malvaceae & 0,57 & Arbusto \\
\hline & Xanthosoma sp. & Zango morado & Araceae & 0,14 & Hierba \\
\hline \multirow{3}{*}{ Folclore } & Hyptis cf sidifolia (L'Hér.) Briq. & Poleo de monte & Lamiaceae & 0,29 & Hierba \\
\hline & Nicotiana tabacum $\mathrm{L}$. & Tabaco & Solanaceae & 0,14 & Arbusto \\
\hline & Sisyrinchium sp. & Planta milagrosa & Iridaceae & 0,14 & Hierba \\
\hline \multirow{14}{*}{$\begin{array}{l}\text { Industriales y } \\
\text { artesanales }\end{array}$} & Oenocarpus bataua Mart. & Ungurahua, kunkuki & Arecaceae & 0,43 & Palma \\
\hline & Cyathea sp. & Helecho arbóreo & Cyatheaceae & 0,29 & Árbol \\
\hline & Erythrina ulei Harms & Porotillo & Fabaceae & 0,43 & Árbol \\
\hline & Guadua angustifolia Kunth & Guadua & Poaceae & 0,43 & Árbol \\
\hline & Mucuna elliptica (Ruiz y Pav.) DC. & Ojo de venado & Fabaceae & 0,29 & Arbusto \\
\hline & Wettinia maynensis Spruce & Teren, huevos de toro & Arecaceae & 0,14 & Palma \\
\hline & Carludovica sp. & Paja toquilla & Cyclanthaceae & 0,29 & Arbusto \\
\hline & Desmoncus sp. & Macaña & Arecaceae & 0,57 & Palma \\
\hline & Iriartea deltoidea Ruiz y Pav. & Ampakai, pambil & Arecaceae & 0,86 & Palma \\
\hline & $\begin{array}{l}\text { Stromanthe stromanthoides (J.F. Mac- } \\
\text { br.) L.Andersson }\end{array}$ & Mamango & Marantaceae & 0,14 & Hierba \\
\hline & $\begin{array}{l}\text { Calathea lutea (Aubl.) E.Mey. ex } \\
\text { Schult. }\end{array}$ & Bijao & Marantaceae & 0,29 & Hierba \\
\hline & Canna indica $\mathrm{L}$. & Achira & Cannaceae & 0,71 & Hierba \\
\hline & Heliconia ortotricha L.Andersson & Heliconia & Heliconiaceae & 0,71 & Hierba \\
\hline & Heliconia vellerigera Poepp. & Platanillo o bijao & Heliconeaceae & 0,29 & Hierba \\
\hline \multirow{17}{*}{$\begin{array}{l}\text { Medicinales, } \\
\text { veterinarias y } \\
\text { tóxicas }\end{array}$} & Aerva sanguinolenta (L.) Blume & Escancel & Amaranthaceae & 0,57 & Hierba \\
\hline & Arracacia sp. & Maya & Apiaceae & 0,14 & Hierba \\
\hline & Brugmansia $\times$ candida Pers. & Guando rosado & Solanaceae & 0,29 & Arbusto \\
\hline & Costus scaber Ruiz y Pav. & Caña agria & Costaceae & 0,29 & Hierba \\
\hline & Cymbopogon sp. & Hierba Luisa & Poaceae & 0,43 & Hierba \\
\hline & Dioscorea sp. & Kenken & Dioscoreaceae & 0,14 & Trepador \\
\hline & Eryngium foetidum $\mathrm{L}$. & Culantrillo & Apiaceae & 0,29 & Hierba \\
\hline & Everardia montana Ridl. & Piripiri & Cyperaceae & 0,43 & Hierba \\
\hline & Helianthus sp. & Amargo & Asteraceae & 0,14 & Arbusto \\
\hline & Heliocarpus americanus L. & Balsilla & Malvaceae & 0,14 & Árbol \\
\hline & Hyptis sp. & Menta natural & Lamiaceae & 0,14 & Hierba \\
\hline & Lantana camara $\mathrm{L}$. & Muras & Verbenaceae & 0,43 & Hierba \\
\hline & Mansoa alliacea (Lam.) A.H.Gentry & Ajo macho & Bignoniaceae & 0,71 & Arbusto \\
\hline & $\begin{array}{l}\text { Ochroma pyramidale } \\
\text { (Cav. ex Lam.) Urb. }\end{array}$ & Balsa & Malvaceae & 0,14 & Árbol \\
\hline & Piper aduncum $\mathrm{L}$. & Matico & Piperaceae & 0,57 & Arbusto \\
\hline & Piper umbellatum $\mathrm{L}$. & Santa María & Piperaceae & 0,57 & Arbusto \\
\hline & Sambucus nigra $\mathrm{L}$. & Tilo & Adoxaceae & 0,29 & Arbusto \\
\hline
\end{tabular}




\begin{tabular}{|c|c|c|c|c|c|}
\hline \multirow{8}{*}{$\begin{array}{l}\text { Medicinales, } \\
\text { veterinarias y } \\
\text { tóxicas }\end{array}$} & $\begin{array}{l}\text { Solanum nigrescens M. Martens y } \\
\text { Galeotti }\end{array}$ & Mortiño & Solanaceae & 0,43 & Hierba \\
\hline & Solanum occultum Bohs & Kukush & Solanaceae & 0,29 & Arbusto \\
\hline & $\begin{array}{l}\text { Stachytarpheta cayennensis (Rich.) } \\
\text { Vahl }\end{array}$ & Rabo de ratón & Verbenaceae & 0,29 & Hierba \\
\hline & Tagetes erecta $\mathrm{L}$. & Flor de muerto & Asteraceae & 0,43 & Hierba \\
\hline & Urera sp. & Ortiga macho & Urticaceae & 0,57 & Hierba \\
\hline & Urera baccifera (L.) Gaudich. ex Wedd. & Chine de monte & Urticaceae & 0,29 & Hierba \\
\hline & $\begin{array}{l}\text { Urera caracasana (Jacq.) Gaudich. ex } \\
\text { Griseb. }\end{array}$ & Sakura & Urticaceae & 0,29 & Arbusto \\
\hline & Vernonanthura patens (Kunth) H.Rob. & Laritaco & Asteraceae & 0,57 & Arbusto \\
\hline
\end{tabular}

*IVUs = Índice de valor de uso; ** = Hábito de Crecimiento

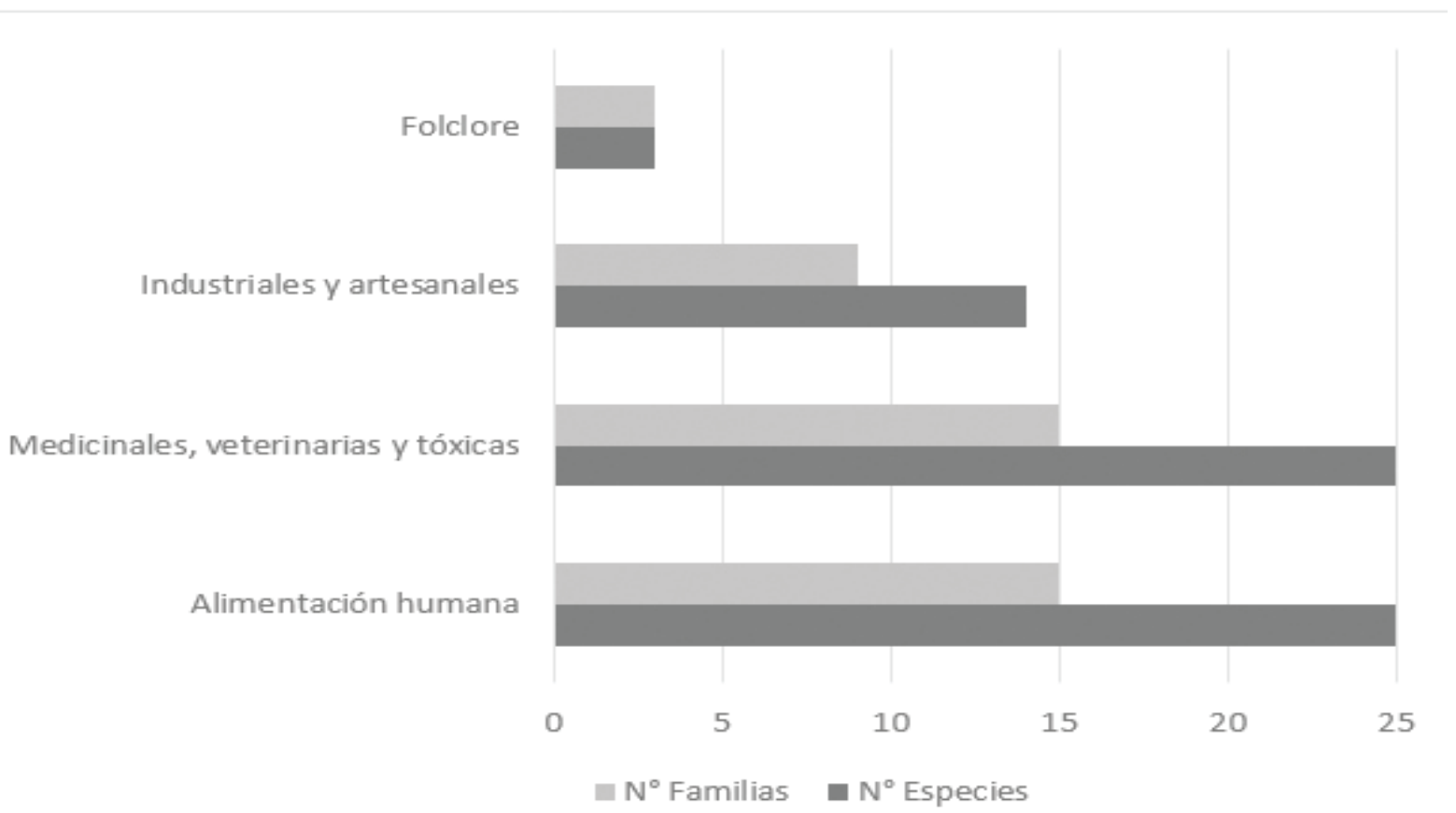

Figura 2. Categorías de usos de los PFNM en las ajas de la parroquia Nankais.

Figure 2. Categories of NTFPs uses in the ajas of Nankais parish.

Los valores de uso (IVUs) más altos corresponden a Psidium guajava (1), Mauritia flexuosa (0,85), Plukenetia volubilis $(0,85)$, Canna indica $(0,71)$ y Heliconia ortotricha $(0,71)$ (Tabla 2$)$. La parte más usada son las hojas, las afecciones más tratadas son dolores estomacales, la aplicación más representativa es el antiséptico para lavar heridas y cicatrizante. Las formas de empleo más comunes son: cocciones, baños, emplastos e infusiones. Se reportaron 25 especies en la alimentación humana, de la cuales principalmente de consumen los frutos y tubérculos y en menor proporción su cogollo (ápice) como las palmas. También se reportaron plantas de usos ancestrales (3) utilizadas en rituales o limpias contra el aire según sus creencias; en cuanto a las artesanías principalmente se utilizan las semillas, tallos y cortezas de las plantas (Figura 2).

Las especies vegetales presentan mayor dominancia con hábito de crecimiento herbáceo (25), esto debido a que son especies que presentan más adaptabilidad, su dispersión y propagación es rápida y son de fácil accesibilidad en cuanto a sus partes vegetativas ya sea para uso medicinal o alimenticio. Consecutivamente predomina el estrato arbustivo (18), arbóreo (15) y Palma (7) (Figura 3). 


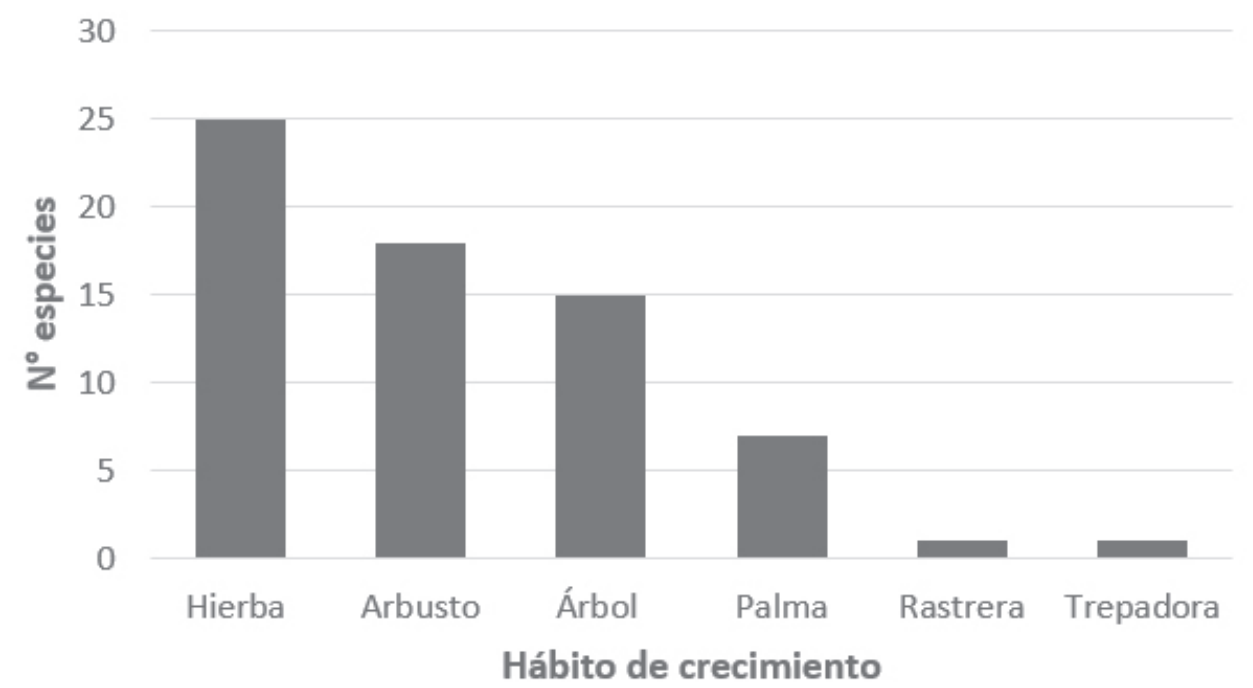

Figura 3. Dominancia del hábito de crecimiento de las especies con PFNM de origen vegetal en las ajas shuar de la parroquia Nankais. Figure 3. Dominance of the growth habit of species with NTFPs of plant origin in the shuar ajas of the Nankais parish.

\subsection{Etnobotánica, derechos de la naturaleza y el buen vivir o sumak kawsay}

Al abordar los derechos de la naturaleza, se parte de la necesidad de reintegrarnos a nosotros mismos en la más amplia comunidad de la Tierra, recordando que el suelo bajo nuestros pies, y bajo las raíces, las patas, las aletas y los tentáculos de aquellos con quienes hemos coevolucionado, es el denominador común que nos unifica. Es lo que nos brinda nuestro hogar compartido, el material del cual estamos hechos y el alimento que nos sustenta (Cullinan, 2019).

Al respecto, las comunidades ancestrales consideran que el garantizar estos derechos les permite tener suelos, aire, agua limpia y paisajes sanos y, por ende, se lograría vivir en armonía con la naturaleza y contar con una vida en plenitud (sumak kawsay); no obstante, lograr aquello implica comprender el funcionamiento del mundo natural en la Tierra y el universo, por ejemplo, el fenómeno de la gravedad se expresa en el alineamiento de los planetas, el crecimiento de las plantas y el ciclo de la noche y el día. Todas son manifestaciones de la existencia de este fenómeno que gobierna el funcionamiento del universo.

Para lograrlo, se requieren nuevas filosofias ecocéntricas del derecho y de la gobernanza, que se materializan en los derechos de la naturaleza. De hecho, esta transformación jurídica ya está en marcha. Por ejemplo, en Ecuador gracias a la cooperación entre pueblos indígenas, organizaciones no gubernamentales y líderes que conciben ancestralmente la naturaleza como Madre Tierra (Pacha Mama), en septiembre de 2008, lograron que la Constitución reconozca a la naturaleza como sujeto de derechos, es decir, tiene derecho a existir, a mantener sus ciclos vitales, su estructura, funciones y procesos.

Asimismo, "la Constitución del Ecuador (2008) y la Constitución Política del Estado de Bolivia (2009) [1o] traducen, respectivamente, por 'buen vivir' y 'vivir bien"' (Hidalgo-Capitán y Cubillo-Guevara, 2014, p. 3). En Suramérica, el Estado Pluricultural de Bolivia incorporó en su Constitución el término en aymara-quechua suma qamaña, traducido como "vivir bien". Por su parte, Ecuador en la Constitución de 2008 marcó una de las pautas que hace hincapié en la inclusión del término kichwa sumak kawsay, "buen vivir".

Una de las ideas que está implícita en las citadas Constituciones garantistas de los derechos de la naturaleza, es el sumak kawsay, que es entendido como una forma de vida en armonía con la naturaleza y con otros seres humanos. Esta idea parte de una concepción de la vida deseable inspirada en la cultura de los pueblos indígenas, quechuas y aymaras especialmente, y se apoya en los principios de equidad social y sostenibilidad ambiental (Hidalgo-Capitán y Cubillo-Guevara, 2014, p. 26).

Esta forma de vida es entendida como plena, equilibrada, sana, armónica y modesta, en los planos individual y social. Es un sistema de vida consciente que mantiene y cultiva relaciones de equilibrio y armonía con la naturaleza que le permite al ser humano alcanzar una existencia en plenitud (Maldonado Ruiz, 2010). En el 
ámbito de la etnobotánica esta ideología se aplica el momento en que se realizan las actividades de recolección y consumo de productos vegetales naturales, las que no deben ocasionar sobreutilización o sobreexplotación, pues desembocarían en una declinación de las poblaciones nativas con fuertes implicaciones ecológicas y económicas.

En este contexto, para fines comprensivos del sumak kawsay, desde una perspectiva de los derechos de la naturaleza, se traen a colación los principios propuestos por Ávila Santamaría (2019), lo cuales son:

- Relacionalidad: denota que todos los miembros de la naturaleza se encuentran vinculados en una red de interacciones interdependientes.

- Reciprocidad: involucra una correspondencia mutua en donde los miembros de la naturaleza toman lo que necesitan y, a la vez, dan lo que requieren, con el fin de asegurar la existencia de todos los miembros.

- Complementariedad y correspondencia: señalan que un miembro de la naturaleza necesita de los otros miembros para existir, se trata de una relación entre miembros distintos que forman parte de una gran unidad.

- Afectividad y espiritualidad: implican el reconocimiento y desarrollo de las distintas emociones y sentimientos que nos permiten establecer formas alternativas de comprender el mundo diferente a las hegemónicas que han instrumentalizado y destruido la naturaleza.

- Ciclicidad y tiempo: hacen referencia a una comprensión en espiral de la temporalidad, según la cual todo avanza y todo cambia y se modifica, pero siempre se regresa al punto inicial, tal como sucede en los ciclos agrícolas.

Con relación al párrafo anterior, al aplicar los principios antes descritos en el caso de estudio a través de los talleres participativos con las informantes, se obtuvo que la relacionalidad se manifiesta en la interdependencia entre las mujeres (shiram nua) con su aja respecto a su manejo, uso y aprovechamiento, es importante señalar que las ajas shuar contienen un sinnúmero de especies que se aprovechan y son vitales para diversas actividades (medicina tradicional, subsistencia y recreacionales) de la nacionalidad shuar.

La reciprocidad se aplica cuando la mujer shuar, como la custodia de su aja, valora primordialmente la vida y la existencia de la naturaleza, ya que manifiesta que no soy parte del aja ni el aja es parte de mí. Somos distintos uno del otro y, sin embargo, ambos somos parte de la misma Pacha Mama, por tanto, se aprovecha elementos vegetales (hojas, tallos, semillas, ápices, exudados y cortezas) necesarios para la subsistencia familiar, al mismo tiempo que se garantiza la sostenibilidad de las especies; así también la complementariedad y la correspondencia se aplican a través de la diversificación de especies, usos y aplicaciones.

Finalmente, la afectividad y la espiritualidad en el aja shuar se refleja en la conservación de especies utilizadas para rituales ancestrales y espirituales; la ciclicidad y el tiempo se aplica en el sistema de agricultura migratoria, ya que se aprovecha durante pocos años; después de este periodo continúa una fase de barbecho (autopoiesis) con la finalidad de recuperar sus ciclos vitales.

\subsection{Manejo del aja shuar mediante conocimientos ecológicos tradicionales}

Las prácticas de manejo ancestral del aja shuar van más allá de una interacción física, ya que se evidencian conexiones subjetivas, las cuales se describen a continuación.

A diferencia de un huerto agrícola de un mestizo, éstas se ubican lejos de las casas. Además, sirve como vivero para luego trasplantar las especies en otros espacios para su crecimiento (propagación vegetal por regeneración natural). Su producción la comparten con grupos de diferentes etnias, no obstante, no es común que los mestizos u otro grupo étnico ingresen a estos lugares que se consideran respetables, ya que éstos irrumpen la armonía del aja y, por ende, afectan a la producción y salud del ecosistema.

Previo a la entrada al aja es costumbre iniciar con un canto ancestral, acompañado del sonido del machete que se golpea contra un fuste; es como una especie de ritual para el ingreso. Los acompañantes deben pisar por donde camina la dueña del aja. A cada paso, revisan las plantas y les hablan en idioma shuar-chicham.

La recolección de los elementos vegetales se realiza únicamente para el consumo familiar y en caso de existir excedentes se realiza un intercambio mediante trueque con otras mujeres de la comunidad. Cada actividad realizada (recolección, siembra y labores silviculturales) en el aja requiere un canto diferente y cuando se encuentra con la piedra sagrada le rinde tributo. Cabe destacar que estos ritos especiales solo pueden ser ejecutados y presenciados por la dueña del aja.

Los cultivos no están ordenados porque conservan la esencia de selva y las plantas se adaptan a cualquier espacio. El suelo está abonado por el desecho de las plantas y por la descomposición de los troncos de los árboles cortados, por tanto, no se evidencia el uso de agroquímicos, fungicidas, herbicidas y variedades híbridas 
de cultivos; valoran más a las variedades nativas y tratan de conservarlas. Para facilitar procesos ecológicos del ecosistema como la polinización y dispersión de semillas, en cada aja shuar, se implementa producción apícola, con especies nativas de abejas.

Para el cuidado de la composición, estructura y función del aja shuar las mujeres lo hacen a través de la especie Sisyrinchium sp, conocida comúnmente como planta milagrosa, ya que éstas le atribuyen propiedades repelentes y alopáticas a plagas, enfermedades fitosanitarias y especies exóticas invasoras.

Según las mujeres shuar en su aja todo tiene sentido y conectividad, por tal motivo un aja tiene tres años de vida útil y luego se busca otro espacio para colocar las mejores semillas y mantenerlas para el alimento familiar. El propósito de este cambio es que el suelo se rehabilite para que produzca mejores frutos y que los bancos de semillas del suelo que se encuentren esperando las condiciones ambientales apropiadas para su germinación logren su cometido.

\section{Discusión}

Las mujeres informantes de la parroquia Nankais son poseedoras de un conocimiento pragmático y perspicaz acerca del uso de las plantas, además, lo practican intensa y frecuentemente, lo que concuerda con Santín Luna (2004), puesto que concluye que la comunidad shuar tiene gran conocimiento sobre la diversidad de usos y beneficios que tiene el bosque, razón por la que tratan de mantener y conservar estos recursos, no siendo igual en la etnia Saraguro, ya que en su caso la población local conoce su uso ancestral, pero el aprovechamiento es ocasional, lo cual provoca la pérdida generacional de conocimientos (Minga et al., 2017).

Continuando con el argumento anterior, al contrastar con la etnia mestiza la tendencia es similar. Así, se reporta que los adultos mayores aún conservan los conocimientos etnobotánicos, pero los aprovechan ocasionalmente, esto implica pérdida de conocimientos en la población juvenil debido al limitado uso y escasa transmisión de saberes generacionales, lo que provoca la merma acelerada de conocimientos ancestrales (Guamán-Songor et al., 2021; López-Beltrán et al., 2021; Quito-Ulloa et al., 2021).

La categoría medicina humana es altamente representativa, debido a la riqueza específica alta (25 especies) similar a la reportada por Guamán et al. (2021); López-Beltrán et al. (2021); Quito-Ulloa et al. (2021) y Marín Gutiérrez et al. (2020); adicionalmente, también se resalta que estas plantas constituyen una manera para efectuar un tratamiento rápido, accesible y económicamente posible, constituyendo así una principal fuente de medicinas para sanar las enfermedades comunes de la etnia, esta tendencia es similar a la reportada por Mora Roman et al. (2017) y Tituaña Pilco y Yánez Orozco (2020).

Otra categoría representativa en cuanto a número de especies y familias botánicas fue alimentos y bebidas, entre estas especies destacan las pertenecientes a la familia Arecaeae, puesto que son para los shuar muy importantes porque constituyen su alimentación diaria; por ejemplo, el meristemo terminal (palmito) y los frutos de la chonta (Cabrera, 1998; Santín Luna, 2004).

La especie Psidium guajava, presentó un IVUs alto, debido a su uso etnobotánico amplio (Rodríguez Amado et al., 2013), ya que se evidenció que esta especie ofrece a la comunidad una amplia gama de usos (comestible, alimento de animales, apícola, combustible, materiales, social y medicinal).

Los hábitos herbáceo y arbustivo, presentaron mayor diversidad específica (riqueza de especies alta), coincidiendo con Jørgensen y León-Yánez (1999), quienes reportaron que los hábitos de hierbas y arbustos son más numerosos en el Ecuador y el órgano más utilizado fue la hoja, no obstante Cerón Martínez (2003) y Minga et al. (2017) en estudios similares con las comunidades Alao y Saraguro reportaron al hábito arbóreo como el más abundante y el tallo la parte botánica usada con mayor frecuencia, seguido por el fruto y en menor proporción las hojas, esto alude a diferencias culturales interétnicas con la comunidad shuar.

Es preciso indicar que para la comunidad shuar de Nankais los derechos de la naturaleza, buen vivir y derechos bioculturales, implica convivir en armonía con todos los seres de la naturaleza (manejo del aja shuar mediante conocimientos ecológicos tradicionales), por converger en un mismo hábitat en el cual ineludiblemente existe un punto de equilibrio, el cual se logra con el desarrollo de un relacionamiento que se basa en el entendimiento y respeto con la naturaleza, así como la comprensión de formas espirituales y cosmológicas que integran al ser humano con la naturaleza y la consecución de mecanismos sofisticados de comunicación (cánticos ancestrales) con seres no humanos.

$\mathrm{Al}$ reportar una alta riqueza de especies (67) en 7 ajas con una superficie de 20 ha y variedad de conocimientos ancestrales, se evidencia un alto respeto hacia los derechos de la naturaleza a su existencia y al man- 
tenimiento y regeneración de sus ciclos vitales, estructura, funciones y procesos evolutivos, aplicabilidad de los principios del sumak kawsay y los derechos bioculturales; esto concuerda con la Ordenanza que establece las áreas de conservación y uso sustentable de la biodiversidad (2019) promulgada por el GAD de Zamora Chinchipe, el cual reconoce al aja shuar como una práctica ancestral de conservación in situ que garantiza la permanencia en el largo plazo del germoplasma utilizado ancestralmente por la nacionalidad shuar, así como los conocimientos ancestrales y prácticas de manejo mediante conocimientos ecológicos tradicionales (cosmológicos y espirituales).

Los conocimientos ancestrales a la luz de los derechos de la naturaleza, sumak kawsay y derechos bioculturales, que se desarrollan en el aja shuar, garantiza la conservación de las especies e identidad cultural, el mantenimiento de la armonía en la naturaleza y el desarrollo de su idiosincrasia en sus interacciones con el hábitat que los rodea, mientras que el desuso por la aculturación, desencadena la pérdida de la biodiversidad en sus niveles: específico, ecosistémico, genético y étnico cultural, así como los derechos de los cuales son titulares las comunidades ancestrales.

Al respecto, Santín Luna (2004) menciona que en Nangaritza existe una gran diversidad de especies, debido a la importancia que le atribuyen los indígenas shuar. Así, éstos son poseedores de un buen conocimiento, especialmente jóvenes, adultos, ancianos y curanderos; debido a que en la actualidad aún se siguen usando mucho las plantas, a pesar de la influencia de la medicina occidental y presencia de centros de salud. No ocurre lo mismo con las comunidades de colonos presentes en este contexto, ya que existe escaso conocimiento de las especies medicinales, debido a que son comunidades que se han asentado hace pocos años en esta zona por procesos de colonización dirigida y semidirigida.

\section{Conclusiones}

El área levantada de 20 ha da cuenta de la existencia de 67 especies utilizadas por las mujeres shuar (sin considerar productos con potencial no estudiados), siendo las categorías más representativas medicinales y alimentación humana, debido a que el aja shuar constituye la principal fuente de medicinas y alimentos de la comunidad.

La aplicación de técnicas de manejo basadas en conocimientos ecológicos tradicionales, las interconexiones establecidas, cercanía y observación de la naturaleza por parte de las mujeres denotan armonía y respeto por los derechos de la naturaleza, sumak kawsay, derechos bioculturales, ciclos vitales de regeneración y autopoiesis.

Las mujeres de la nacionalidad shuar de Nankais, logran conservar sus paisajes sanos mediante la aplicación de un manejo tradicional de sus ajas, que va más allá de una interacción física, puesto que se establecen conexiones subjetivas (respeto, cantos ancestrales y conversaciones con los elementos bióticos y abióticos), las cuales denotan un éxito, similar o mayor a los propuestos por la cultura occidental, ya que se evidencia una alta diversidad de especies, mantenimiento de la composición, estructura y función (procesos ecológicos) de los ecosistemas.

\section{Contribuciones de los autores}

- Héctor Rolando Zhiñin Quezada: conceptualización, investigación, metodología, recursos, redacción, borrador original, revisión y edición

- $\quad$ Bladimir Valentin Poma Mendoza: Análisis formal, Validación.

- Leonardo Paúl González Nivelo: Curación de datos.

- Gonzalo Bladimir Quito Ulloa: Recursos, Análisis formal.

\section{Referencias}

Aguirre, Z. (2012). Guía para estudiar los productos forestales no maderables. Documento para estudiantes de la Carrera de Ingeniería Forestal. Universidad Nacional de Loja.

Aij', J. (1984). Pueblo de fuertes: rasgos de historia shuar para los planteles biculturales de educación media. Ediciones Abya Yala: Sistema de educación radiofónica bicultural shuar.

Ávila Santamaría, R. (2019). La utopía del oprimido. Los derechos de la naturaleza y el buen vivir en el pen- 
samiento crítico, el derecho y la literatura. Edicionesakal.

Balcázar Calle, R. A. (2016). Investigación de la pintura corporal shuar y su significado, para la creación de un manual y sus aplicaciones gráficas. Escuela Superior Politécnica de Chimborazo.

Cabrera, C. (1998). Identificación de árboles y arbustos silvestres con uso alimenticio en la provincia de Zamora Chinchipe. Universidad Nacional de Loja.

Cerón Martínez, C. E. (2003). Etnobotánica quichua del río Yasuní, Amazonía ecuatoriana. Cinchonia, 4(1), 1-20. https://revistadigital.uce.edu.ec/index.php/CINCHONIA/article/view/2314

Cogollo Calderón, A. M., \& García-Cossio, F. (2012). Caracterización etnobotánica de los productos forestales no maderables (PFNM) en el corregimiento de Doña Josefa, Chocó, Colombia. Revista Biodiversidad Neotropical, 2(2), 102-112. https://doi.org/10.18636/bioneotropical.v2i2.70

Constitución de la República del Ecuador [Const]. 20 de octubre de 2008.

Cullinan, C. (2019). El derecho salvaje. Universidad Andina Simón Bolívar, Sede Ecuador

Figuera Vargas, S. C., \& Cujilema Quinchuela, K. C. (2017). El sumak kawsay desde la perspectiva del sistema jurídico ecuatoriano. Justicia, 23(33), 51-70. https://doi.org/10.17081/just.23.33.2882

Gálvez, M. (2010). Guía Puyo. Guía comercial, profesional y turística de Pastaza. Culturas indígenas de Pastaza. Indigenas shuar. http://www.guiapuyo.com/index.php

Gobierno Autónomo Descentralizado [GAD] Nankais. (2019). Plan de desarrollo y ordenamiento territorial de Nankais.GAD Nankais

Grijalva, J., Ramos, R., Vera, R., Barrera, P, \& Sigcha, F. (eds.) (2013). Primer Encuentro de Bosques, Recursos Genéticos Forestales y Agroforestería. Memorias del Evento. Instituto Nacional Autónomo de Investigaciones Agropecuarias.

Guamán-Songor, J., Peña-Tamayo, J., Jaramillo-Díaz, N., \& Granda Pardo, J. (2021). Productos forestales no maderables de origen vegetal en cinco comunidades rurales del cantón Palanda, provincia de Zamora Chinchipe, Ecuador. Bosques Latitud Cero, 11(1), 43-56. https://revistas.unl.edu.ec/index.php/bosques/article/view/901

Hidalgo-Capitán, A. L.-, \& Cubillo-Guevara, A. P. (2014). Seis debates abiertos Buen vivir: sobre secuestros, domesticaciones, rescates y alternativas. Íconos. Revista de Ciencias Sociales, 48, 25-45. https://doi. org/10.17141/iconos.48.2014.1204

Instituto Nacional de Investigaciones Agropecuarias [INIAP]. (2008). Estado de los recursos fitigenéticos para la agricultura y alimentación. INIAP, Estación Experimental Santa Catalina.

Jørgensen, P. M., \& León-Yánez, S. (eds.). (1999). Catalogue of the vascular plants of Ecuador. Monogr. Syst. Bot. Missouri Botanical Garden 75. http://www.mobot.org/mobot/research/ecuador/welcomesp.shtml

López-Beltrán, J., Urgilés, N., \& Aguirre Padilla, N. (2021). Productos forestales no maderables de origen vegetal en cinco comunidades de la parroquia Zumba, cantón Chinchipe, provincia de Zamora Chinchipe. Bosques Latitud Cero, 11(1), 28-42. https://revistas.unl.edu.ec/index.php/bosques/article/view/923

Maldonado Ruiz, L. (2010). Interculturalidad y políticas públicas en el marco del Buen Vivir. En G. Fernandez-Juárez (ed.), Salud, Interculturalidad y Derechos.Claves para la reconstrucción del Sumak Kawsay-Buen Vivir (pp. 81-89). Ediciones Abya-Yala.

Marín Gutiérrez, I., Allen-Perkins Avendaño, D., \& Hinojosa Becerra, M. (2020). El documental etnográfico-participativo "Natem, la bebida sagrada de los shuar". Gazeta de Antropología, 31(1), 10. https://doi. org/10.30827/digibug. 36670

Minga, S. R., Jaramillo Díaz, N., \& Aguirre Mendoza, Z. (2017). Productos forestales no maderables de origen vegetal de cinco comunidades del cantón Yacuambi, Zamora Chinchipe. Bosques Latitud Cero, 7(1), 7289. https://revistas.unl.edu.ec/index.php/bosques/article/view/173

Mora Roman, G., Quizhpe Coronel,W., Castro, P., \& Jadan, O. (2017). Conocimiento sobre productos forestales no maderables en dos pisos florísticos: piemontano y montano bajo, en bosque semi-caducifolio, Santa Rufina, Loja, Ecuador. Bosques Latitud Cero, 7(2), 39-54. https://revistas.unl.edu.ec/index.php/ bosques/article/view/320

Morales Valverde, R., Tardío, J., Aceituno-Mata, L., Molina, M., \& Pardo de Santayana, M. (2011). Biodiversidad y etnobotánica en España. Memorias de la Real Sociedad Española de Historia Natural, 9(2), 157-207. http://digital.csic.es/handle/10261/66932

Oficina Internacional del Trabajo [OIT]. (2007). Boletín 2007. La OIT y los pueblos indígenas tribales. Tema: Discrimación. (Pro 169). OIT. https://www.ilo.org/wcmsp5/groups/public/@ed_norm/@normes/documents/publication/wcms_100544.pdf 
Ordenanza que establece las áreas de conservación y uso sustentable de la biodiversidad en la provincia de Zamora Chinchipe. 09 de enero de 2019. http://zamora-chinchipe.gob.ec/wp-content/uploads/2019/03/71.ORDENANZA-QUE-ESTABLECE-AREAS-DE-CONSERVACION-Y-USO.pdf

Quito-Ulloa, G., Quito-Ulloa, M., Urgiles-Gómez, N., \& Aguirre-Mendoza, Z. (2021). Productos forestales no maderables de origen vegetal de la parroquia Valladolid, cantón Palanda, provincia de Zamora Chinchipe. Bosques Latitud Cero, 11(1), 1-14. https://revistas.unl.edu.ec/index.php/bosques/article/view/916

Reátegui Encarnación, K. G. (2011). Shuar aja saberes y prácticas ancestrales. Universidad de las Regiones Autónomas de la Costa Caribe Nicaraguense (URACCAN).

Rodríguez Amado, R., Lafourcade Prada, A., \& Pérez Rondón, L. (2013). Hojas de Psidium guajava L. Revista Cubana de Farmacia, 47(1), 127-135. http://scielo.sld.cu/scielo.php?script=sci_abstract\&pi$\mathrm{d}=\mathrm{S} 0034-75152013000100014 \& \operatorname{lng}=\mathrm{es} \& \mathrm{nrm}=\mathrm{iso} \& \mathrm{tlng}=\mathrm{en}$

Santín Luna, F. M. (2004). Ethnobotany of the Communities of the upper rio Nangaritza. Lyonia, 7(2), 105-122.

Tituaña Pilco, M. E., \& Yánez Orozco, E. F. (2020). Análisis florístico y etnobotánico de la comunidad Shuin Mamus-taisha, Amazonía del Ecuador. Universidad Estatal Amazónica. 